Rev Biomed 2006; 17:45-60.

\title{
Síndrome de envenenamiento paralizante por consumo de moluscos.
}

Revisión

Martha L. Hernández-Orozcoํㅛ Ismael Gárate-Lizárraga² .

${ }^{1}$ Instituto Mexicano del Seguro Social. ${ }^{2}$ Centro Interdisciplinario de Ciencias Marinas (CICIMAR-IPN). Departamento de Plancton y Ecología Marina. La Paz, B.C.S., México.

\section{RESUMEN.}

El envenenamiento paralizante por consumo de moluscos (EPM) es un síndrome neurotóxico asociado a la presencia de saxitoxina (STX) y sus más de 26 análogos en los mariscos marinos. Las toxinas son producidas por diversas especies de dinoflagelados del género Alexandrium, Pyrodinium bahamense var. compresum y Gymnodinium catenatum, así como en algunas especies de cianobacterias. La sintomatología ocasionada por la saxitoxina y sus derivados se centra principalmente en el sistema nervioso, ya que al bloquear los canales de sodio produce parálisis; puede ser tan leve como adormecimiento de labios hasta la muerte por falla ventilatoria. El diagnóstico debe realizarse clínicamente y corroborando la toxina por medio de un bioensayo en ratón o el aislamiento en los organismos productores. No existe antídoto alguno, el tratamiento es sintomático enfocado a mantener la mecánica ventilatoria. En nuestro país se han observado brotes tóxicos en ambos litorales, sin embargo, existen pocos programas de inspección y monitoreo. La incidencia de episodios tóxicos, así como la presencia de toxinas paralizantes se ha incrementado y se ha reportado en lugares en donde antes no era común por esta razón, consideramos importante el divulgar este tipo de intoxicaciones, ya que representan un gran riesgo para la salud humana. (Rev Biomed 2006; 17:45-60)

Palabras clave: envenenamiento paralizante por consumo de moluscos, Saxitoxina, toxinas marinas, Gymnodinium catenatum, Pyrodinium bahamense var. compressum.

\section{SUMMARY.}

Paralytic poisoning syndrome due to the shellfish consumption.

Paralytic shellfish poisoning (PSP) is a neurotoxic syndrome related to shellfish containing saxitoxin (STX) and its more than 26 analogues. Toxins are produced by several species of dinoflagellates from the genera Alexandrium, Pyrodinium bahamense var. compressum, Gymnodinium catenatum and some species of cianobacteria. The symptoms caused by the saxitoxin and its derivatives mainly attack the nervous system. Diagnosis of this paralytic shellfish poisoning must be done by means of mouse bioassay or isolating the toxin producing organisms. There is no antidote and the treatment for intoxication is symptomatic, focused on maintaining the ventilator mechanism. In our country, toxic episodes have been observed in both littoral shores; however, there are

Solicitud de sobretiros: Ismael Gárate-Lizárraga, CICIMAR-IPN, Departamento de Plancton y Ecología Marina, Avenida Politécnico s/n, Col. Playa Palo de Santa Rita, C.P 23096, La Paz, B.C.S., México. Correo electrónico: igarate@ipn.mx Recibido el 22/Junio/2005. Aceptado para publicación el 8/Noviembre/2005.

Este artículo está disponible en http://www.uady.mx/sitios/biomedic/revbiomed/pdf/rb061717.pdf 


\section{Hernández-Orozco, I Gárate-Lizárraga.}

few monitoring programs. The incidence of toxic episodes as well as the presence of paralytic toxins has increased and they have been reported in new areas where they were previously uncommon. For this reason it is important to divulge information about this kind of poisoning since it represents a potential danger to human health. (Rev Biomed 2006; 17:45-60)

Key words: paralytic shellfish poisoning, Saxitoxin (PSP), marine toxins, Gymnodinium catenatum, Pyrodinium bahamense var. compressum.

\section{INTRODUCCIÓN.}

Las microalgas marinas son plantas microscópicas unicelulares que forman la base de la cadena alimenticia en el mar. Bajo condiciones favorables, pequeñas poblaciones algales pueden multiplicarse rápidamente y formar densos florecimientos constituidos de millones de células por litro. Los florecimientos pueden colorear el agua y comúnmente son llamados "mareas rojas", sin embargo, no todos los florecimientos algales son rojos, ya que su coloración (amarilla, café, naranja, verde, etc.) dependerá de los pigmentos presentes en las microalgas responsables de tales florecimientos. Muchos florecimientos o mareas rojas son causados por especies no tóxicas. En algunas ocasiones no es necesaria una proliferación masiva de las especies para que estas representen un peligro real para la salud humana. De las más de 2000 especies estimadas de dinoflagelados, alrededor de 75 especies tiene la capacidad de producir potentes toxinas y causar intoxicaciones en humanos y unas cuantas son potencialmente letales $(1,2)$.

Los fenómenos conocidos como florecimientos algales nocivos (FAN o HABs por sus siglas en inglés: Harmful Alage Blooms) han cobrado una connotación alarmante a nivel mundial, debido al aparente incremento de la frecuencia, intensidad y distribución geográfica de estos episodios; resultado del aumento del conocimiento que se tiene del tema, de la acuacultura, de los nutrientes o del transporte de quistes en el lastre de barcos. Muchos de estos florecimientos algales son inocuos y contribuyen grandemente a la fertilidad de las zonas costeras, sin embargo algunos tienen efectos negativos sobre los niveles tróficos superiores (3-5). Entre los impactos nocivos se encuentran la muerte de organismos por taponamiento de sus branquias, por la creación de condiciones hipóxicas ó anóxicas (con poca o nula concentración de oxígeno), así como la acumulación de toxinas en diversos órganos de organismos marinos. Esto último ocasiona intoxicaciones de seres humanos por consumo de productos marinos (moluscos o peces) contaminados con las toxinas generados por diversas especies del fitoplancton, particularmente de dinoflagelados y diatomeas (6-13).

Los principales grupos de venenos producidos por las microalgas, descritos hasta la fecha y que afectan directamente al hombre son: veneno paralizante de molusco (VPM), veneno diarreico de molusco (VDM), ciguatera o veneno ciguatérico de pescado (VCP), veneno amnésico por consumo de mariscos (VAM), veneno neurotóxico (VN) y microcistinas, éstas últimas producidas por algas verde-azuladas (cianobacterias) en aguas salobres y lacustres. Estos compuestos pueden causar varios tipos de envenenamientos, los cuales se encuentran resumidos en el cuadro 1 (14-24). No obstante, el número de toxinas que se descubren y su sintomatología va en incremento día con día. $(25,26)$.

Se ha supuesto que probablemente el registro más antiguo de los efectos tóxicos de microalgas aparece en el Antiguo Testamento (Éxodo 719-21; 1291 A.C.), donde se señala que "las aguas del río Nilo en Egipto se transformaron en sangre produciendo la muerte masiva de peces y su descomposición". El primer caso de intoxicación humana por consumo de mariscos tóxicos que está registrado, se produjo el 15 de junio de 1793, en la costa oeste de los Estados Unidos y es descrito por el capitán George Vancouver en "A Voyage of Discovery to the North Pacific Ocean, and Round the World" publicado en 1798 (27).

Históricamente, los episodios de envenenamientos por consumo de moluscos (EPM) en aguas marinas han sido asociados principalmente con 11 especies

\section{Revista Biomédica}




\section{Envenenamiento paralizante por consumo de moluscos.}

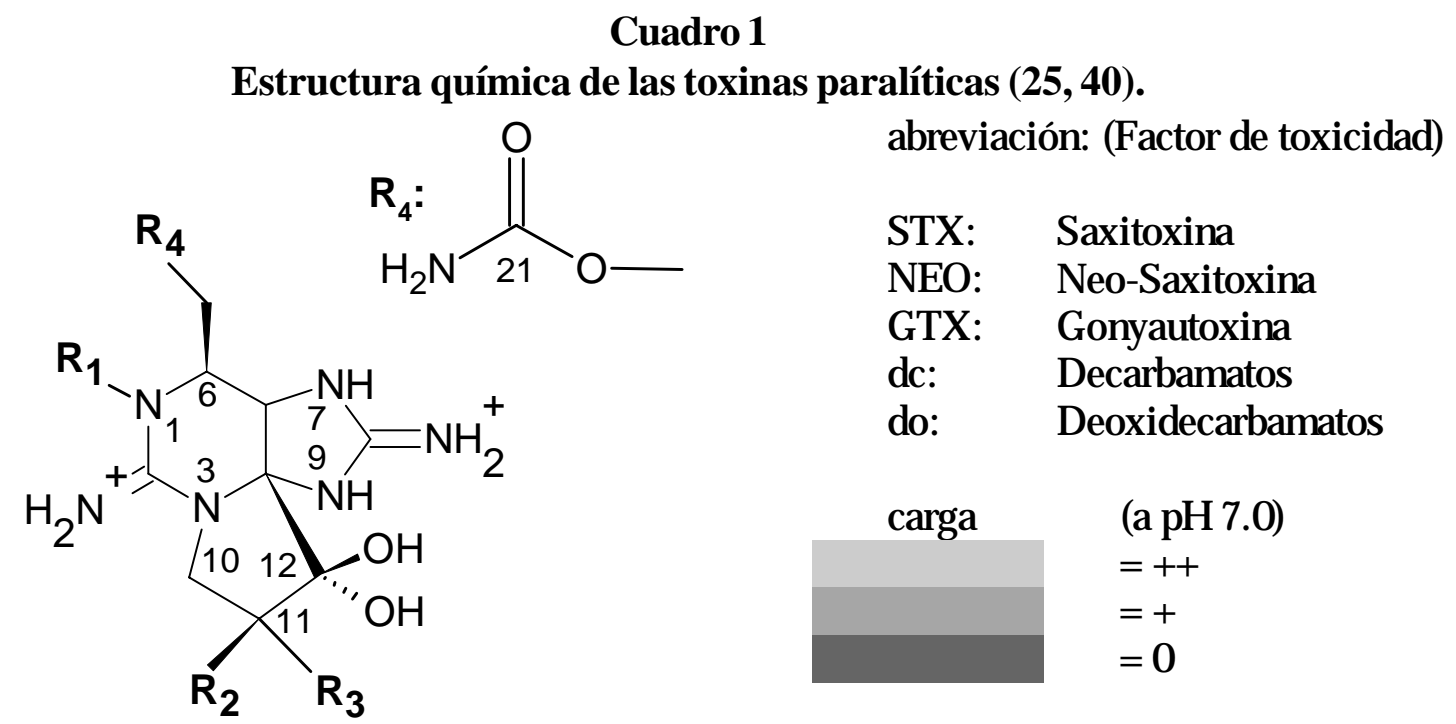

\begin{tabular}{|c|c|c|c|c|c|c|}
\hline & & & $\begin{array}{c}\text { toxinas } \\
\text { carbamatos }\end{array}$ & $\begin{array}{c}\text { toxinas } \\
\text { N-Sulfocarbamatos }\end{array}$ & $\begin{array}{c}\text { toxinas } \\
\text { Decarbamatos }\end{array}$ & $\begin{array}{l}\text { Toxinas } \\
\text { Deoxidecarba } \\
\text { matos }\end{array}$ \\
\hline $\mathbf{R}_{\mathbf{1}}$ & $\mathbf{R}_{\mathbf{2}}$ & $\mathbf{R}_{\mathbf{3}}$ & $\mathbf{R}_{4}:$ OCO-NH & $\mathrm{R}_{4}: \mathrm{OCONH}^{-\mathrm{SO}_{3}}{ }^{-}$ & $\mathbf{R}_{\mathbf{4}}: \mathbf{O H}$ & $\mathbf{R}_{\mathbf{4}}: \mathbf{H}$ \\
\hline $\mathrm{H}$ & $\mathrm{H}$ & $\mathrm{H}$ & STX & $\mathrm{B}_{1}$ & dcSTX & DoSTX \\
\hline $\mathrm{OH}$ & $\mathrm{H}$ & $\mathrm{H}$ & NEO & $\mathrm{B}_{2}$ & dcNEO & - \\
\hline $\mathrm{OH}$ & $\mathrm{OSO}_{3}^{-}$ & $\mathrm{H}$ & $\mathrm{GTX}_{1}$ & $\mathrm{C}_{3}$ & $\mathrm{doGTX}_{1}$ & - \\
\hline $\mathrm{H}$ & $\mathrm{OSO}_{3}^{-}$ & $\mathrm{H}$ & $\mathrm{GTX}_{2}$ & $\mathrm{C}_{1}$ & $\mathrm{dcGTX}_{2}$ & doGTX $_{2}$ \\
\hline $\mathrm{H}$ & & $\mathrm{OSO}_{3}^{-}$ & $\mathrm{GTX}_{3}$ & $\mathrm{C}_{2}$ & $\mathrm{doGTX}_{3}$ & $\operatorname{doGTX}_{3}$ \\
\hline $\mathrm{OH}$ & $\mathrm{H}$ & $\mathrm{OSO}_{3}^{-}$ & $\mathrm{GTX}_{4}$ & $\mathrm{C}_{4}$ & $\mathrm{doGTX}_{4}$ & - \\
\hline
\end{tabular}

de dinoflagelados del género Alexandrium; sin embargo, los brotes de EPM pueden deberse también a Pyrodinium bahamenses var. compresum, Gymnodinium catenatuam y a diversas especies de cianobacterias tales como Anabaena circinalis, Aphanizomenon flos-aquae, Cylindrospermopsis raciborskii y Lyngbya wollei, las cuales han causado considerables muertes humanas y problemas de salud pública (28-30). Actualmente, la mayoría de las muertes de seres humanos por brotes de EPM o de otros eventos de moluscos tóxicos causados por dinoflagelados, ocurren porque no hay programa local o nacional de monitoreo (14-31). Las toxinas delEPM no son sólo acumuladas por bivalvos filtradores, también se ha documentado que se pueden encontrar en otros organismos, como cangrejos, gasterópodos, macarela y peces planctívoros $(14,32)$.

\section{ETIOLOGÍA.}

En 1927, investigadores de la Universidad de California observaron un florecimiento de dinoflagelados alrededor de un lecho de mejillones, a la vez que gente sufrió parálisis y murió después de comer los mejillones. Los investigadores sospecharon que el dinoflagelado encontrado (Alexandrium catenella) era venenoso, ya que el agua extraída de ellos y de los mejillones mataban ratones de la misma manera. La causa del EPM es la ingestión de una o más toxinas paralíticas. No existe otra forma de contacto conocida que pueda causar intoxicación (33). Sin embargo, los incidentes de EPM seguidos de la ingestión de ciertos cangrejos de arrecifes de coral y ciertos gasterópodos, han sido reportados en Japón y en las Islas Fiji (28). En Indonesia, hubo un incidente que involucró 191 víctimas y 4 muertes, en las que la comida responsable fue un pez 
ML Hernández-Orozco, I Gárate-Lizárraga.

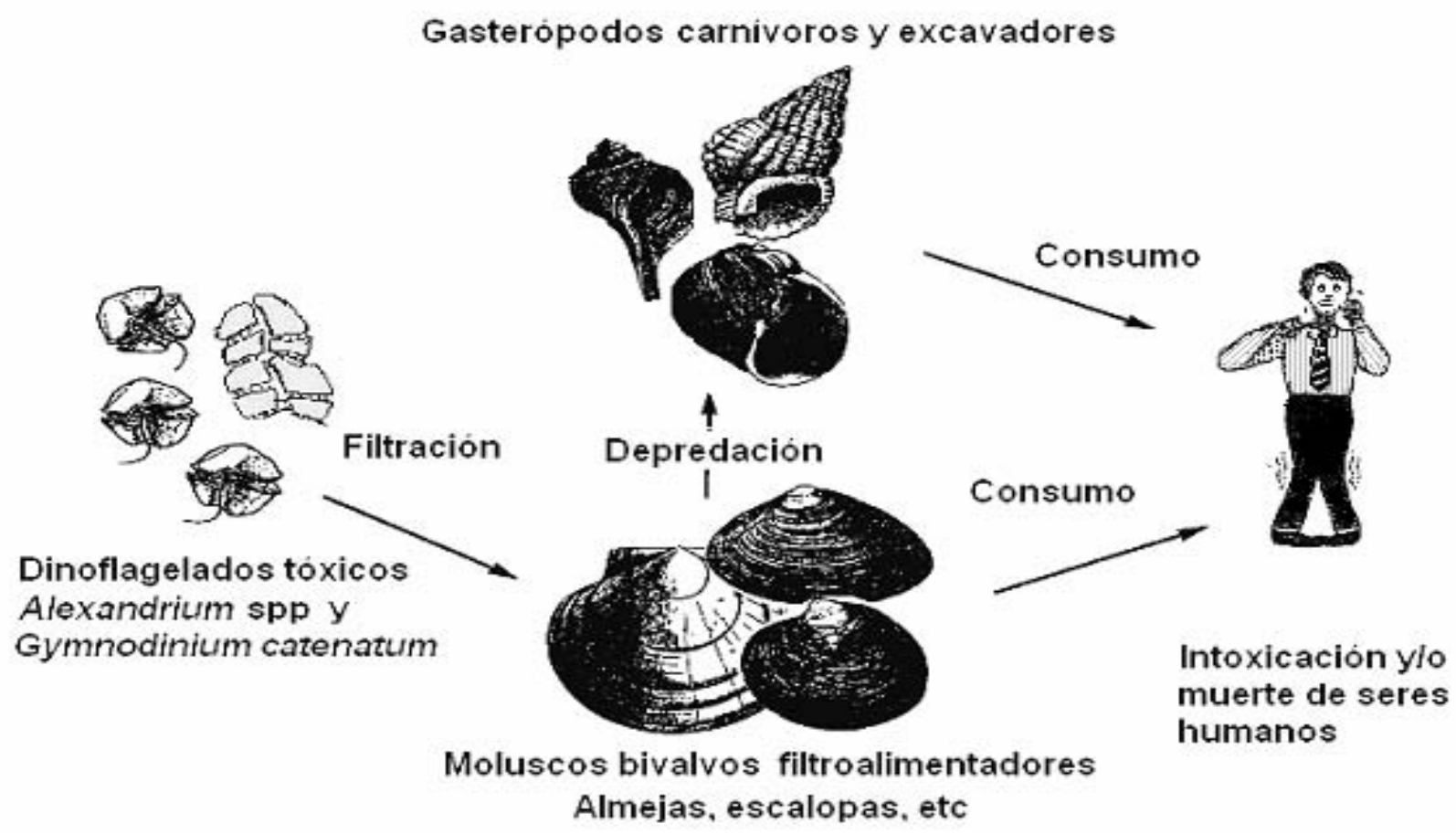

Figura 1.- Escenario que ilustra como las toxinas paralizantes pueden ser acumuladas por los moluscos, llegan a causar la intoxicación de los seres humanos. (Modificado de 34).

cupleiforme. Este es el único episodio en que la intoxicación fue posterior a ingesta de pescado (14).

Los moluscos bivalvos se alimentan filtrando partículas suspendidas en el agua, y entonces transfieren tales partículas de comida de las branquias a los órganos digestivos. Si un florecimiento de dinoflagelados tóxicos ocurre, haya o no discoloración del agua, los moluscos de esa área sirven para concentrar las toxinas paralizantes (34) (figura 1). Los moluscos no son afectados por las toxinas paralizantes, porque muchos de ellos tienen nervios y músculos que trabajan con canales de calcio, mientras que la SXT y otras toxinas paralíticas bloquean sólo canales de sodio $(1,35)$.

\section{TOXICOLOGÍA.}

\section{Efecto de las toxinas paralizantes.}

Las toxinas paralizantes alteran específicamente el transporte del ion sodio, pues son capaces de unirse fuertemente a la proteína de membrana denominada canal de sodio que está presente en casi todas las células de mamíferos, aves, peces y anfibios, y también en invertebrados, como los moluscos bivalvos. Así, el bloqueo de los canales de sodio daña el funcionamiento celular, inhibiendo las señales eléctricas que mantienen trabajando toda nuestra actividad nerviosa superior, vegetativa y la comunicación sináptica (figura 2).

En ausencia de apoyo respiratorio intensivo, la muerte ocurre por parálisis respiratoria, compromiso cardíaco y del sistema nervioso central $(1,28,36)$. La SXT es la principal toxina paralítica, pertenece a la familia de neurotoxinas solubles en agua y es de las más potentes toxinas conocidas. Las saxitoxinas son absorbidas rápidamente a través de la mucosa oral y gastrointestinal. La dosis letal mínima es de $9 \mu \mathrm{g} / \mathrm{kg}$, siendo más potente que el cianuro de sodio, cuya dosis letal mínima es de 10,000 $\mu \mathrm{g} / \mathrm{kg}$. La severidad de los síntomas y la progresión dependen del tamaño de la dosis, susceptibilidad del individuo y del grado de eliminación de las toxina, la cual es excretada principalmente en la orina (37). Desde el punto de vista de su estructura química, las toxinas paralíticas son derivados imidazol guanidínicos con un anillo

\section{Revista Biomédica}




\section{Envenenamiento paralizante por consumo de moluscos.}

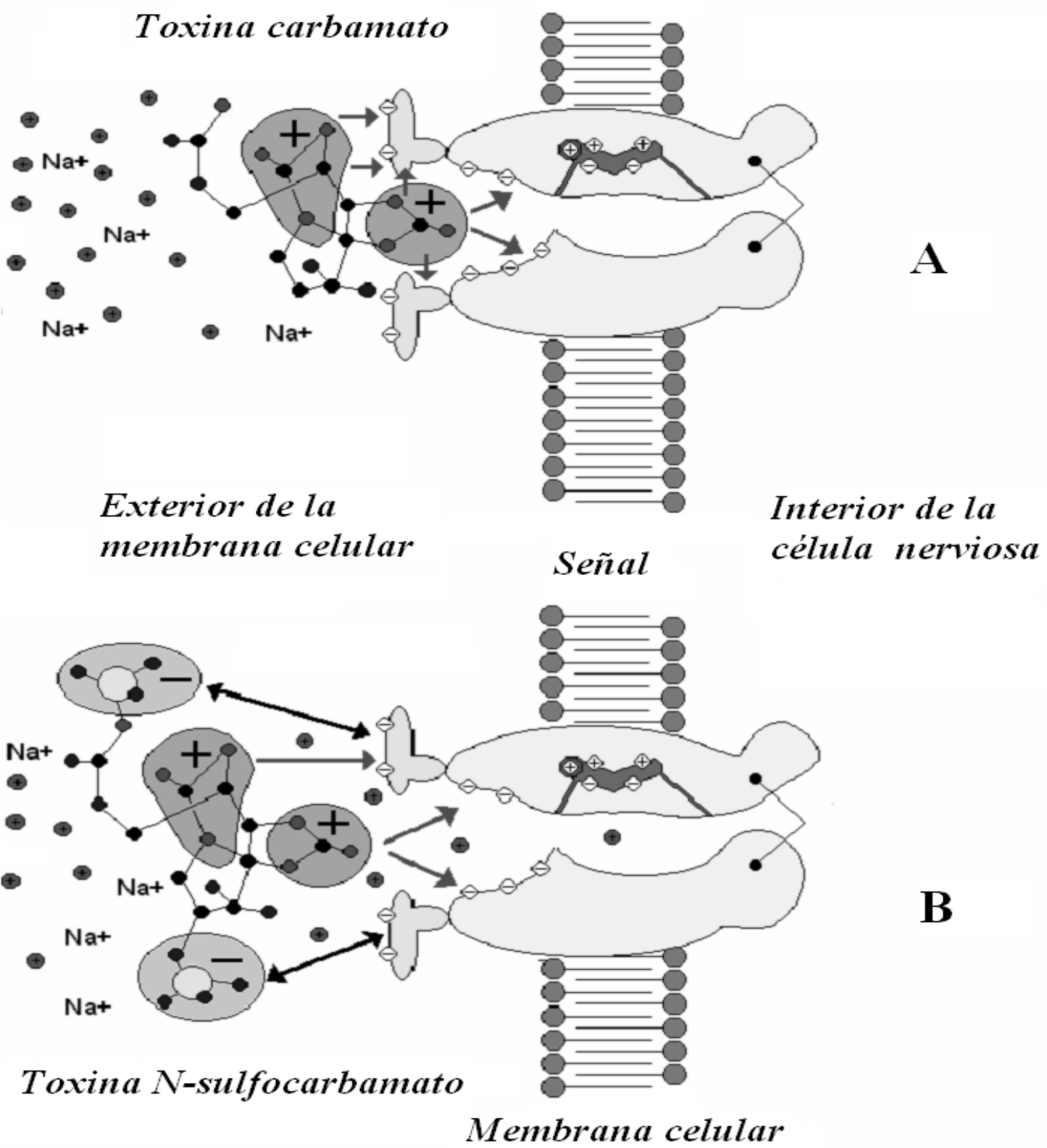

Figura 2.- Mecanismos de acción de toxinas paralizantes, donde se muestra el bloqueo de los canales de sodio a nivel de membrana celular por las toxinas A); toxinas carbamatos y B) toxinas N-sulfocarbamatos (Cortesía de Christian Hummert, Eurofins/Wiertz-Eggert-Jörisse).

fusionado en posición angular y con varios posibles radicales substitutos que tienen más de 24 toxinas paralizantes. Estructuralmente, en función de los radicales, se pueden establecer 4 grupos: toxinas carbamato, toxinas $\mathrm{N}$-sulfocarbamatos, toxinas decarbamatos y deoxicarbamatos. De acuerdo con el número de cargas de la molécula se dividen en toxinas del grupo de la saxitoxina (STX), toxinas del grupo de las gonyautoxinas (GTX) y toxinas del grupo de las C. La toxicidad absoluta de las toxinas paralíticas varía considerablemente (cuadro 2) (25, 38-40).

La mayor parte de los países que realizan control de toxinas VPM en los mariscos utilizan el bioensayo en ratón de la Asociación de Químicos Analíticos Oficiales (AOAC). Este ensayo se había 
ML Hernández-Orozco, I Gárate-Lizárraga.

\section{Cuadro 2}

Principales tipos de envenenamientos, su sintomatología y especies productoras. * *registros en el Pacifico Mexicano $(8,21,22,46,48,49,91,92,94,96,101)$; **registros en las costas mexicanas del Golfo de México $(23,24)$.

\begin{tabular}{|c|c|c|}
\hline $\begin{array}{l}\text { Tipo de toxinas y de } \\
\text { Envenenamientos }\end{array}$ & Sintomas generales & Especies responsables \\
\hline $\begin{array}{l}\text { PARALIZANTES } \\
\text { Envenenamiento } \\
\text { Paralizante por } \\
\text { consumo de Moluscos } \\
\text { (EPM; PSP). }\end{array}$ & $\begin{array}{l}\text { Sensación de hormigueo, Entumecimiento } \\
\text { de cara cuello, manos, náuseas, vómito y } \\
\text { muerte por paro respiratorio }\end{array}$ & $\begin{array}{l}\text { Gymnodiniumcatenatum }(*) \\
\text { Alexandriumacatenella }(*) \\
\text { Alexandrium catenella }(*) \\
\text { Alexandriumminutum }(*) \\
\text { Alexandriumtamarensis }(*) \\
\text { Alexandrium tamiyavanichii }(*) \\
\text { Alexandriumspp } \\
\text { Pyrodiniumbahamense v. compressum } \\
(*) \\
\text { Cianobacterias }\end{array}$ \\
\hline $\begin{array}{l}\text { DIARREICAS } \\
\text { Envenenamiento } \\
\text { Diameico por consumo } \\
\text { de Moluscos } \\
\text { Abrev: (EDM; DSP). }\end{array}$ & $\begin{array}{l}\text { Diarrea, náuseas, vómito y la exposición } \\
\text { crónica promueve la formación de } \\
\text { tumores en el sistema digestivo }\end{array}$ & $\begin{array}{l}\text { Dinophysis acuminata }(*) \\
\text { Dinophysis caudata }(*, * *) \\
\text { Dinophysis. norvegica }(*) \\
\text { Dinophysis fortii }(*, * *) \\
\text { Dinophysis sacculus }(*, * *) \\
\text { Dinophysis miles }(*) \\
\text { Dinophysis spp } \\
\text { Prorocentrumlima }(*, * *) \\
\text { Prorocentrumspp } \\
\end{array}$ \\
\hline $\begin{array}{l}\text { AMNESICAS } \\
\text { Envenenamiento } \\
\text { Amnésico por consumo } \\
\text { de Moluscos } \\
\text { Abrev: (EAM; ASP). }\end{array}$ & $\begin{array}{l}\text { Síntomas gastrointestinales como: } \\
\text { vómito, diarrea y calambres. Síntomas } \\
\text { neurológicos: Desorientación, náuseas, } \\
\text { vértigo, confusión y pérdida temporal de } \\
\text { la memoria. }\end{array}$ & $\begin{array}{l}\text { Pseudo-nitzschia australis }(*,) \\
\text { Pseudo-nitzschia pungens var. multiseries } \\
\text { Pseudo-nitzschia seriata }(*, * *) \\
\text { Amphora coffaeiformi } \\
\text { Nitzschia navis-varingica }\end{array}$ \\
\hline $\begin{array}{l}\text { NEUROTOXICAS } \\
\text { Envenenamiento } \\
\text { neurotóxico } \\
\text { Abrev: (EN; NP). }\end{array}$ & $\begin{array}{l}\text { Escalofríos, dolor de cabeza, debilidad } \\
\text { muscular, náuseas, vómito y muerte por } \\
\text { paro respiratorio }\end{array}$ & $\begin{array}{l}\text { Karenia brevis }(*, * *) \\
\text { Karenia selliformis } \\
\text { Karenia bidigitata } \\
\text { Karenia spp. }\end{array}$ \\
\hline $\begin{array}{l}\text { CIGUATERICAS } \\
\text { Envenenamiento } \\
\text { Ciguaténico por } \\
\text { consumo de Pescado } \\
\text { Abrev: (ECP; CFP) }\end{array}$ & $\begin{array}{l}\text { Náuseas, entumecimiento y temblor de las } \\
\text { manos y los pies, vómito y en casos } \\
\text { extremos, muerte por fallas respiratorias }\end{array}$ & $\begin{array}{l}\text { Gambierdiscus toxicus }(*, * *) \\
\text { Ostreopsis spp }(*, * *) \\
\text { Coolia spp }(*, * *)\end{array}$ \\
\hline
\end{tabular}

aplicado con éxito durante varias décadas como único método reconocido internacionalmente para determinar las toxinas con fines regulatorios. El nivel máximo permisible para su consumo o comercialización, es de $80 \mu \mathrm{g} / 100 \mathrm{~g}$ de carne fresca y de acuerdo con el método de bioensayo en ratones es de $400 \mathrm{UR} / 100 \mathrm{~g} / \mathrm{tejido.} \mathrm{Una} \mathrm{UR} \mathrm{es} \mathrm{la}$ cantidad de toxina inyectada, la cual podría matar a 1 ratón de $20 \mathrm{~g}$ en 15 minutos y es equivalente a
$0.18 \mu \mathrm{g}$ de STX (41). Además del bioensayo en ratón, existen otros métodos para identificar y cuantificar saxitoxinas: se utilizan cromatografía líquida de alta resolución (HPLC, por sus siglas en inglés: high performance liquid cromatography), inmunoensayo por enzimas, determinaciones electrofisiológicas, métodos fluorimétricos y bloqueo de receptores $(27,34-42)$.

\section{Revista Biomédica}




\section{Envenenamiento paralizante por consumo de moluscos.}

\section{EPIDEMIOLOGÍA.}

Los reportes de enfermedades transmisibles en USA muestran que de 1985 a 2003 hubo 22 casos de EPM por 100000 habitantes y ningún deceso(54). Se considera que representan cerca del $1.1 \%$ de las enfermedades por alimentos, y el $7.4 \%$ de las intoxicaciones por productos marinos en $\mathrm{EU}$ (55). A nivel mundial, alrededor de 2,000 casos de intoxicaciones humanas son reportadas por año, con una tasa de mortalidad del 15\% (3). Además de las intoxicaciones humanas, el EPM ha sido implicado en la muerte de aves marinas y ballenas jorobadas, las cuales se alimentaron en aguas donde ocurrió una marea roja (56).

La incidencia de EPM en humanos, hasta 1970, había reportado 1600 casos de intoxicación registrados en el mundo, principalmente en Norteamérica y Europa. Desde entonces cerca de 900 casos adicionales han sido reportados, ocurriendo en regiones donde VPM era desconocido $(26,57,58)$. En Guatemala en el verano de 1987, 187 personas fueron afectadas por VPM, 26 de ellas fallecieron, la mortalidad en niños mayores de 6 años fue $50 \%$ mientras que en adultos fue de 7\% (59). Mientras que un estudio realizado en Alaska se encontró que de 117 casos (de 1973 a 1992), el 25\% requirió de intubación, falleciendo sólo una persona, por lo que la tasa de mortalidad es alrededor del $8.5 \%$. Sin embargo, en países desarrollados esta tasa de mortalidad es menor del 1\% (60). En 1974, la incidencia fue estimada en alrededor de 1600 casos, con más de 300 muertes al año. En 1996 en la isla Kodiak (Alaska) se estableció una incidencia de 15 muertes por 1000 personas intoxicadas al año (61).

En México, uno de los primeros casos sobre la intoxicación por consumo de moluscos ocurrió en Mazatlán, Sinaloa en $1979(62,63)$. A la fecha este envenenamiento es uno de los más peligrosos, causando un porcentaje aproximado del $72 \%$ de los brotes de envenenamiento por comida marina que ha ocurrido en la última década. Tres de estos eventos representan $87 \%$ de los casos de envenenamiento (460 individuos intoxicados, 32 muertes), en Mazatlán, Guerrero y Oaxaca, las áreas más afectadas (64-66) (SSA, PROFEPA). Por otra parte, un gran número de organismos marinos (peces y tortugas) también son afectados por los brotes de EPM $(2,52,66-71)$. En la figura 3 se presentan la distribución de Gymnodinium catenatum, Pyrodinium bahamense var. compressum dos especies productoras de toxinas paralíticas, así como de las áreas donde se ha reportado la presencia de dichas toxinas.

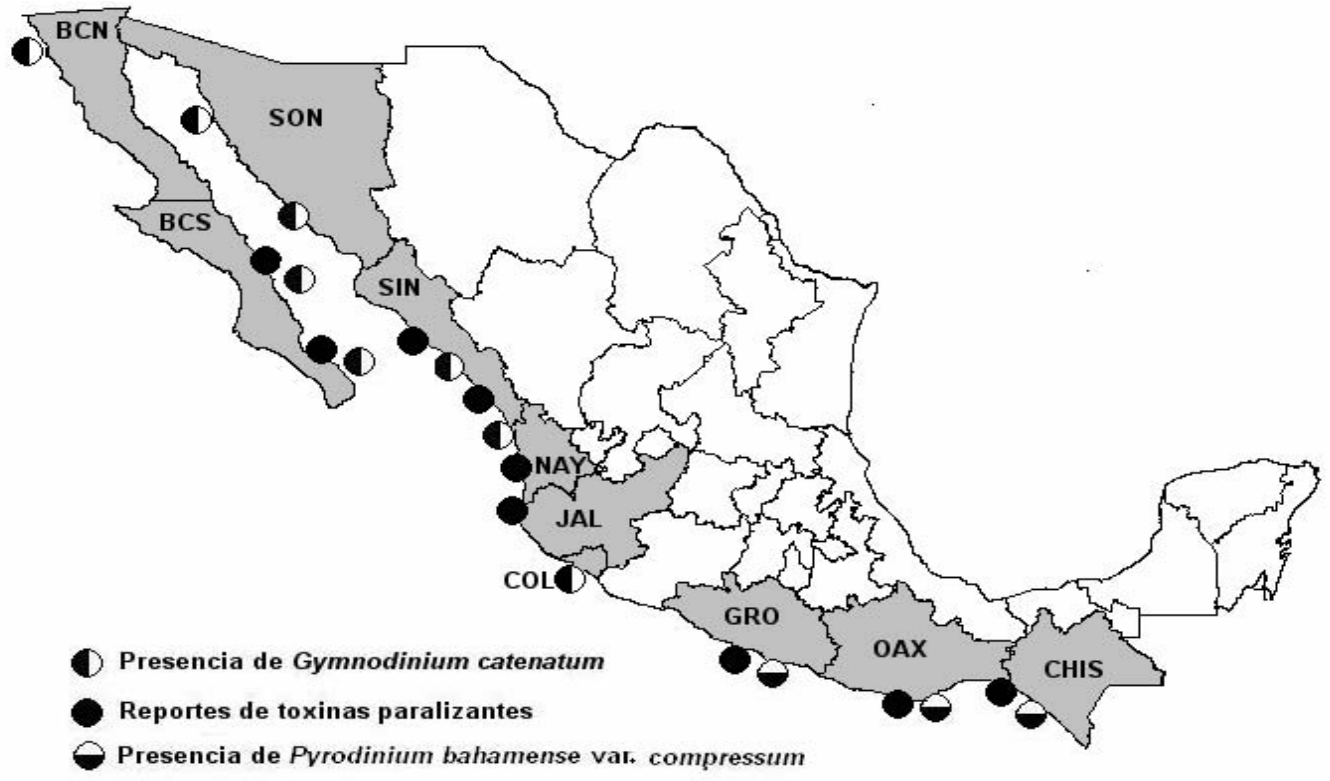

Figura 3.- Distribución de dos especies productoras de toxinas paralizantes y de la presencia de reportes de estas toxinas en moluscos bivalvos $(62-71,90-96,101)$.

Vol. 17/No. 1/Enero-Marzo, 2006 


\section{Hernández-Orozco, I Gárate-Lizárraga.}

El control de las biotoxinas marinas es difícil, las enfermedades no pueden prevenirse por completo. Todas las toxinas son de naturaleza no proteica y extremadamente estables. Así, el cocinado, ahumado, secado o salado no las destruye, y no puede predecirse por el aspecto de la carne del pescado o de los productos pesqueros, si el alimento es o no tóxico. La principal medida preventiva es la inspección y muestreo de las zonas de pesca y de los bancos de moluscos bivalvos o poblaciones de gasterópodos, así como el análisis de las toxinas. El bioensayo en ratones se utiliza a menudo para este propósito y se realiza una determinación confirmatoria por la técnica de HPLC $(25,36,72)$, si la muerte sobreviene después de 15 minutos. Si se encuentran niveles altos de toxinas, se interrumpen las capturas comerciales de moluscos, principalmente $(44,73)$. La eliminación de toxinas mediante técnicas de depuración puede tener cierto potencial, pero el proceso es lento y costoso. En Japón y Corea se usan arcillas pulverizadas (montmorillonita) para flocular las microalgas y llevarlas al fondo del mar, esto tiene un impacto ecológico, pero se considera de menor riesgo que la toxicidad del florecimiento algal (27).

\section{CUADRO CLÍNICO Y TRATAMIENTO.}

Los síntomas del EPMinician aproximadamente de 30 minutos a 3.3 horas de consumir el molusco contaminado. Los primeros síntomas que se presentan son: hormigueo, pesantez y adormecimiento de lengua y labios. Estos efectos son claramente debidos a la absorción local de las toxinas EPM a través de la membrana de la mucosa oral. Esta sensación se esparce a la cara, cuello. El hormigueo en dedos de manos y pies es frecuente, así como un moderado dolor de cabeza y mareo. Algunas veces náusea y vómito ocurren en etapas tempranas; aunque las bases del vómito en EPM no son bien conocidas. En una epidemia en el norte de Inglaterra, cerca de la tercera parte de las victimas presentó vomitó (14).

En los casos de envenenamiento moderado, las parestesias progresan hacia los brazos y las piernas, acompañadas de debilidad motora (cuadro 3). Así mismo existe el vértigo y discurso incoherente. Una sensación de flotar y ligereza son frecuentes. La base de este disturbio tampoco es clara, pero es probablemente atribuido a alguna interferencia con señales propioceptivas aferentes como la conducción de impulsos en nervios sensoriales con mecanismos centrales. Manifestaciones cerebelosas como ataxia, incoordinación motora y dismetría son frecuentes. Los reflejos pueden estar ausentes o normales y la mayoría de los pacientes están calmados y conscientes. Otros efectos del sistema nervioso autónomo que pueden ocurrir son salivación, taquicardia y diaforesis $(74,75)$. La dificultad respiratoria comienza a aparecer con la sensación de constricción en la garganta.

En envenenamientos severos, la parálisis muscular se extiende y es más profunda; puede presentarse una acidosis láctica de mecanismo desconocido como manifestación temprana (cuadro 3). La muerte puede ocurrir dentro de 2 a 24 horas

\section{Cuadro 3}

Síntomas clínicos causados por EPM (1)

\begin{tabular}{|l|l|l|}
\hline Intoxicación leve & Intoxicación moderada & Intoxicación severa \\
\hline Niveles de STX 80-120 $\mu \mathrm{g}$ & $120-180 \mu \mathrm{g}$ & $300-1200 \mu \mathrm{g}$ \\
Náusea y vómito & Insuficiencia respiratoria & Pupilas dilatadas no reactivas \\
Debilidad muscular & Parálisis músculos faciales & Distonía profunda \\
Parestesia en labios & Reflejo nauseoso ausente & Parálisis respiratoria \\
Parestesia en extremidades & Lengua inmóvil & Hipotensión \\
inferiores & & Paro cardiaco \\
\hline
\end{tabular}

\section{Revista Biomédica}




\section{Envenenamiento paralizante por consumo de moluscos.}

dependiendo de la dosis y es atribuible al decremento progresivo de la eficiencia ventilatoria y al gradual incremento de hipoxia, hipercapnia y todo lo asociado con desequilibrio fisiopatológico (76). El pulso no muestra anormalidades, en algunos pacientes que se han hospitalizado se ha encontrado elevación de su tensión arterial y esto se ha correlacionado a la dosis de saxitoxina ingerida (77).

Los efectos cardiovasculares de la SXT son debidos a la resistencia periférica. El corazón no se afecta directamente, no hay mucha contribución del sistema nervioso central, cuando las toxinas VPM, son absorbidas a través del tracto gastrointestinal, se distribuyen uniformemente en el agua corporal. Los efectos vasculares periféricos consisten en una vasodilatación por efecto de la musculatura vascular, así como de una disminución del tono vasomotor, seguida de un bloqueo de nervios vasoconstrictores; además la STX bloquea los nervios vasoconstrictores antes que los nervios vasodilatadores. Por lo anterior, la STX está entre los agentes hipotensores más potentes, $2-3 \mu \mathrm{g} / \mathrm{kg}$ inyectados vía intravenosa producen una caída de la presión arterial de cerca del $50 \%$ del nivel normal (38); con una dosis menor a 1 $\mu \mathrm{g} / \mathrm{kg}$, la STX tiene un efecto hipertensivo como respuesta compensatoria a la acción de vasodilatación. En individuos intoxicados con dosis pequeñas de análogos de la STX y si el paciente sobrevive 24 horas, el pronóstico es bueno, pero la debilidad muscular puede persistir por semanas.

El diagnóstico de la intoxicación se basa en el antecedente de reciente ingesta de comida marina, principalmente moluscos bivalvos (78). Los hallazgos físicos están enfocados en disfunción neural o neuromuscular con insignificantes manifestaciones cardiovasculares. La muerte es atribuida a la parálisis parcial de los músculos respiratorios, y cuando está presente, significa moderado o severo envenenamiento, requiriendo cuidado hospitalario (cuadro 3).

Los resultados obtenidos en estudios de la conducción nerviosa en pacientes con EPM muestran un alargamiento de la latencia, disminución de la velocidad de conducción y una moderada disminución de la amplitud en nervios periféricos motores y sensoriales (79-81).

El VPM no se asocia con el nivel de toxina, método de preparación, dosis, raza, sexo, edad. El consumo de alcohol ha sido visto como factor de protección por algunos autores (60). Existen reportes de elevación de la enzima cinasa creatina de fracción MB (82-83), pero no han sido confirmados por otros estudios como para considerarse un criterio diagnóstico y existe la sospecha de que fue efecto de otras toxinas asociadas a la STX.

\section{Diagnóstico diferencial.}

El envenenamiento causado por los pesticidas de anticolinesterasa puede ser un diagnóstico diferencial difícil, especialmente si las víctimas del envenenamiento de anticolinesterasa no son vistas hasta que la fase inicial del estímulo colinérgico ha pasado. Las manifestaciones gastrointestinales como nausea, arqueo, vómito y diarrea, pueden ser confundidas por expresiones similares en EPM. Pero en el envenenamiento por anticolinesterasa los síntomas gastrointestinales se mejoran después de administrar atropina. Otra evidencia es la estimulación colinérgica, como salivación excesiva, lagrimeo y secreción bronquial, así como miosis pupilar; esto ayuda a diferenciarla del EPM. En la intoxicación por pesticidas anticolinesterasa, la parálisis muscular resulta de una acumulación de acetilcolina y como resultado da la desensibilización de receptores colinérgicos nicotínicos (14).

El envenenamiento por consumo de pez globo es otra forma de envenenamiento por comida marina que debe ser diferenciado del EPM. El agente responsable es la tetradotoxina y el inicio es agudo, generalmente progresivo y dominan las manifestaciones neurológicas virtualmente idénticas al EPM. Aunque la alta incidencia de esta intoxicación ocurre en Japón, hay casos esporádicos en otras regiones. La toxina responsable es químicamente diferente a las toxinas de EPM, pero tienen una acción común ya que ambas bloquean los canales de sodio de nervios, músculos y otras membranas excitables. La incidencia de nauseas y vómito es más alta que en 


\section{Hernández-Orozco, I Gárate-Lizárraga.}

EPM, hay siempre un grado de hipotensión. El diagnóstico diferencial está basado en el antecedente de haber comido pez globo $(14,74)$.

El botulismo, envenenamiento causado por la toxina producida por la bacteria Clostridium botulinum, también provoca parálisis flácida. El diagnóstico diferencial no es difícil, principalmente porque el periodo de incubación es más largo (días) que el de VPM. La lesión farmacológica específica es en el nervio activado por la liberación de acetilcolina en todas las uniones colinérgicas y hay evidencia de extensa falla colinérgica $(14,76)$.

La ciguatera, es una intoxicación que ocurre como resultado del consumo de pescado más que de moluscos, sin embargo es necesario descartarla. Como la saxitoxina, la ciguatoxina afecta los canales de sodio, pero en el caso de la ciguatoxina la permeabilidad se aumenta. La parestesia (caracterizada por sensaciones anormales en la piel) es el signo clínico predominante en la ciguatera, la diferencia con EPM puede ser difícil en pacientes que han consumido una mezcla de comida marina (57).

No existe una prueba diagnóstica de laboratorio, sin embargo, si existe alguna sospecha el examen de agua para identificar la presencia de algas tóxicas, pruebas de laboratorio pueden dar evidencia la toxicidad. No hay inmunidad al EPM y el segundo ataque puede ser más severo que el primero. Otros diagnósticos diferenciales menos comunes son: poliradiculoneuropatia aguda, Síndrome de Fisher, miastenia gravis y la parálisis periódica (84); otro diagnóstico aún más raro, es la intoxicación por Oliva vidua fulminans, un molusco marino que provoca sintomatología similar al PSP y que también puede ocasionar la muerte por falla ventilatoria (85).

\section{Tratamiento.}

No hay antídoto específico, aunque se ha experimentado en ratas el uso de la 4 aminopiridina (un bloqueador de canal de potasio), que cuando es administrada a organismos con paro respiratorio, puede acelerar la recuperación aunado a ventilación artificial y tratamiento con bicarbonato de sodio, logrando revertir efectos respiratorios y de la presión arterial, así como reducción de la mortalidad (35). El bloqueo presináptico de canales de potasio da un alargamiento del potencial de acción y retrasa el flujo de iones potasio, permitiendo una mayor entrada de calcio en terminales presinápticas; el aumento de calcio libre provoca liberación de acetilcolina disponible en la unión neuromuscular. Esta información no ha sido avalada por ensayos clínicos $(1,57)$.

El tratamiento clínico es sintomático y está enfocado a mantener la mecánica ventilatoria y tratar de eliminar la toxina. Si el vómito no se ha presentado espontáneamente, hay que inducirlo o realizar un lavado gástrico para eliminar la fuente de toxina no absorbida. Como las toxinas de VPM son resistentes al pH gástrico, éstas pueden ser adsorbidas por el carbón activado, la dosis adulto es $1 \mathrm{~g} / \mathrm{kg}$ de peso $(50 \mathrm{a} 100 \mathrm{~g}$ ) en niños mayores de 2 años $1 \mathrm{~g} / \mathrm{kg}$ peso (15 a $30 \mathrm{~g}$ ) y debe administrarse en los primeros 30 minutos de ingerido el molusco contaminado. Estos pasos son especialmente importantes en el manejo de niños victimas de este tipo de envenenamiento, como la severidad de la intoxicación es directamente dependiente de las concentraciones en el cuerpo (57, 74).

En caso de intoxicación moderada o grave, mantener una adecuada ventilación es el principal objetivo. En VPM no complicadas la vía área no es obstruida por excesiva secreción. La falla ventilatoria es por parálisis de nervios y músculos respiratorios, la asistencia respiratoria con presión positiva es recomendable. Un monitoreo periódico de $\mathrm{pH}$ sanguíneo y gases es importante para asegurarse de una adecuada oxigenación, especialmente en vista de que hallazgos en animales de experimentación muestran que la STX produce acidosis láctica. Los fluidos son esenciales para corregir la posible acidosis, y también facilitar la excreción renal de la toxina. No existe razón para la aplicación de agentes anticolinesterasa para mejorar el funcionamiento muscular, tampoco hay bases para recomendar un ejercicio vigoroso ya que en una defectuosa ventilación (y posiblemente circulación) sólo puede incrementar la producción de lactato (75).

La vida media de eliminación de la STX del

\section{Revista Biomédica}




\section{Envenenamiento paralizante por consumo de moluscos.}

cuerpo humano es de 90 minutos, 9 horas puede ser adecuado en la mayoría de los casos para la reducción fisiológica de las concentraciones de toxina a niveles menos nocivos, excepto en aquellos casos que la concentración llegue a ser excepcionalmente alta, $\mathrm{o}$ en víctimas con deterioro en la función renal. En los exámenes postmorten no se aprecian anormalidades patológicas, a excepción de la condición pulmonar: los pulmones están edematosos, congestionados y crepitantes al tacto. Posteriormente, se identifica la(s) toxina (s) en el contenido gástrico y en todos los fluidos del cuerpo y muestras de tejido analizadas, con el fin de determinar el perfil de toxinas que provocaron su muerte $(86,87)$. Recientemente se ha utilizado la saxitoxina con fines terapéuticos, como anestésico corneal (88) o como relajante del tono anal (89).

En México, los estudios sobre las toxinas paralizantes son relativamente escasos y esporádicos, aunque cada vez más se incrementan los estudios concernientes en identificar a las especies productoras de este tipo de toxinas, así como los análogos presentes. La primer especie productora de toxinas paralizante que fue identificada y reportada en México fue Gymnodinium catenatum (90). Sin embargo, nuevos registros de especies productoras de toxinas paralíticas en estas costas del Pacífico Mexicano se han incrementado: Alexandrium catenella, A. minutum, A. tamiyavanichi y Pyrodinium bahamense var. compressum (70, 91-94). Se han realizado estudios para conocer el perfil de toxinas en muestras de G. catenatum y en moluscos bivalvos colectados en diversas localidades de la zona costera del golfo de California (95). El perfil de toxinas estuvo compuesto por 11 análogos, destacando la presencia de SXT, NeoSTX, dcSTX, dcGTX2, dcGTX3, B1, B2, C1, C2, C3 y C4. En lo que respecta a la determinación del perfil de toxinas en cepas de Gymnodinium catenatum de diferentes localidades del Golfo de California se encontró que las cepas de Mazatlán y de la Bahía de La Paz son más tóxicas que las de Bahía Concepción, debido a la presencia de neosaxitoxina (NeoSTX) (95-96). En algunas áreas como en Bahía de La Paz, los niveles de toxinas están muy por debajo del límite permisible para consumo humano, mientras que en Bahía Concepción estos valores pueden dispararse hasta los 300 $\mu g$ STXeq/100g molusco (70-91, 94). Por lo que los estudios de monitoreo son necesarios para conocer la variación anual de las especies productoras de toxinas, la concentración de las mismas, así como su perfil de análogos presentes. En un estudio reciente sobre la composición especifica del fitoplancton, que comprende una serie de tiempo de más de 20 años las costas del Golfo de México (23), no se reportan especies productoras de toxinas paralizantes como G. catenatum y ninguna especie del género Alexandrium ni casos oficiales de EPM.

\section{CONCLUSIÓN.}

Los productos marinos son importantes nutricional y económicamente hablando, en Europa 12 billones de libras de productos de la pesca son consumidos anualmente. En Estados Unidos y en Canadá se consumen entre 6 y $7 \mathrm{~kg}$ per capita de alimentos proveniente del mar, mientras que en México, el consumo nacional de productos marinos se ha incrementado, siendo de $10.16 \mathrm{~kg}$ per capita de consumo humano directo, y de $4.30 \mathrm{~kg}$ en el humano indirecto, con un total de $14.46 \mathrm{~kg}$ (97). Por otra parte, en México la producción almejera, principal vector de las toxinas paralizantes, se ha ido incrementando en los últimos años, siendo los principales estados productores: Baja California, Baja California Sur, Sinaloa, Veracruz y Campeche (98). La legislación ayuda a asegurar la calidad y seguridad de los productos, prohibiendo las especies tóxicas, muestreando los niveles de toxina y monitoreando y controlando las importaciones (99). Es por ello que deben de realizarse monitoreos continuos de las especies productoras de toxinas, así como de la determinación de las concentraciones de toxinas en las almejas de importancia comercial en ambas costas de México, lo cual puede prevenir futuros casos de intoxicaciones. Se han descrito mutaciones en moluscos bivalvos que producen variación en su capacidad de acumular toxinas como consecuencia de una adaptación genética a las toxinas paralíticas, este fenómeno puede actuar como agente de selección 


\section{Hernández-Orozco, I Gárate-Lizárraga.}

natural resultando en resistencia de toxina en poblaciones de almejas, pero incrementando el riesgo de EPM en seres humanos (100). Si bien en México existen series de tiempo de más de 15 años de estudios en la mareas rojas $(92,101)$, son pocos los Centros de Investigación o Dependencias Gubernamentales que se realizan monitoreos continuos de fitoplancton tóxico y de biotoxinas marinas. En nuestro país existe la Norma Oficial Mexicana, sin embargo, hay un desconocimiento general por parte de los habitantes de zonas costeras, e inclusive el personal médico no logra reconocer a tiempo la sintomatología característica del EPM, por lo que hemos considerado necesario que ese conocimiento sea difundido. El fenómeno "marea roja" debe ser reconocido por la población, reportado y monitoreado por las autoridades competentes antes de permitir el consumo humano de los organismos vectores de EPM, para evitar brotes de envenenamiento. Algunos estados de la República Mexicana, entre ellos Baja California Sur, ya cuentan con programas de contingencia sobre mareas rojas y biotoxinas marinas, el cual está bajo la coordinación de la Secretaria de Salud del Estado y está conformado por diversos especialistas en taxonomía del fitoplancton, en especial de las especies productoras de toxinas, médicos, cooperativistas de la zona pesquera, así como de especialistas en el análisis de toxinas paralizantes, diarreicas y amnésicas.

\section{AGRADECIMIENTOS.}

Los autores agradecen al Instituto Politécnico Nacional el apoyo para la realización del presente trabajo a través de los proyectos CGEPI 20040626; CGPI2005-0143. MLHO agradece al IMSS, La Paz, B.C.S., por las facilidades otorgadas para la realización de este trabajo. IGL es becario COFAA y EDI. Un agradecimiento especial a María Clara Ramírez Jáuregui (ICMyL-UNAM, Mazatlán) por facilitarnos la literatura especializada.

\section{REFERENCIAS.}

1.- Lehane L. Paralytic Shellfish Poisoning: A review. National Office of Animal and Plant Health, Agriculture, Fisheries and Forestry - Australia, Canberra; 2000.
2.- Anderson DM, Andersen P, Bricelj VM, Cullen JJ, Rensel JE. Monitoring and Management Strategies for Harmful Algal Blooms in Coastal Waters. APEC · 201-MF-01.1. Asia Pacific Economic Program, Singapore, an Intergovernmental Oceanographic Commission Technical Series $N^{\circ}$ 59, Paris 2001.

3.- Hallegraeff GM. A review of harmful algal blooms and their apparent global increase. Phycologia 1993; 32: 79-99.

4.- Van Dolah FM. Marine algal toxins: origins, health effects, and their increased occurrence. Environ Health Perspect. 2000; 108 Suppl 1:133-41.

5.- Brett MM. Food poisoning associate with biotoxins and shellfish. Curr Opin Infect Dis 2003; 16: 461-5.

6.- Lagos N. Micoalgal blooms: a global issue with negative impact in Chile. Biol Res 1998; 31:375-86.

7.- Rhodes FA, Mills CG, Popei K. Paralytic shellfish poisoning in Papua New Guinea. P N G Med J 1975;18: 197 202.

8.- Popkiss ME, Horstman DA, Harpur D. Paralytic shellfish poisoning. A report of 17 cases in Cape Town. Afr Med J 1979; 55: 1017-23.

9.- Tan CT, Lee EJ. Paralytic shellfish poisoning in Singapore. Ann Acad Med Singapore 1986; 15: 77-9

10.- Shumway SE, Barter J, Sherman-Caswell S. Auditing the impact of toxic algal blooms on oysters. Environ Audit 1990; 2: 41-56.

11.- Shumway SE, Cembella AD. The impact of toxic algae on scallop culture and fisheries. Rev Fish Sci 1993; 1: 12150 .

12.- Parrilla-Cerrillo MC, Vázquez-Castellanos JL, SaldateCastañeda EO, Nava-Fernández LM. Brotes de toxiinfecciones alimentarias de origen microbiano y parasitario. Salud Pública Mex 1993; 35: 456-63.

13.- Fleming LE, Katz D, Bean JA, Hammond R. Epidemiology of seafood poisoning. En Hui YH, Kitts D, Stanfield PS editores: Foodborne Disease Handbook Volume 4: Seafood and Environmental Toxins. New York: Marcel Dekker Inc; 2000. p. 297-310.

14.- Kao CY. Paralytic shellfish Poisoning. En Falconer I, editor. Algal Toxins in Seafood and Drinking Water. London: Academic Press; 1993. p. 75-86.

\section{Revista Biomédica}




\section{Envenenamiento paralizante por consumo de moluscos.}

15.- Holmes MJ, Teo SL. Toxic marine dinoflagellates in Singapore waters that cause seafood poisonings. Clin Exp Pharmacol Physiol 2002; 29:829-36.

16.- Sobel J, Painter J. Illnesses caused by marine toxins. Clin Infect Dis 2005; 41: 1290-6.

17.- Ade P, Funari E, Poletti R. Risk to human health associated with marine toxic algae. Ann Ist Super Sanita 2003; 39:53-68.

18.- Gárate-Lizárraga I. Florecimientos algales nocivos. En: Anguas-Vélez B. H. (ed.) Perspectivas para el desarrollo acuacultural del sistema lagunar de Bahía MagdalenaAlmejas, B.C.S., México: Comité Editorial del IPN; 2005. p. $41-48$.

19.- Gómez-Aguirre S, Santoyo-Reyes H. Plancton de lagunas costeras: XI Transporte en tres estuarios del noroeste de México (noviembre 1973). Rev Lat Amer Microbiol 1975; 17 : 175-83.

20.- Licea-Durán S, Gómez-Aguirre S, Cortés-Altamirano R, Gómez S. Notas sobre algunos florecimientos algales y la presencia de especies tóxicas en cinco localidades del Pacífico Mexicano (1996-1999). En: Tresierra-Aguilar AE, Culquichicón-Malpica ZG (editores). VIII Congreso Latinoamericano sobre Ciencias del Mar (COLACMAR), 1721 de octubre de 1999. Libro de resúmenes ampliados, T. 1. Trujillo-Perú. P335-7.

21.- Hernández-Becerril DU. Species of the planktonic diatom genus Pseudonitzscia of the Pacific coast of Mexico. Hidrobiologia 1992; 379: 77-84.

22.- Okolodkov Y, Gárate-Lizárraga I. An Annotated checklist of marine dinoflagellate (Dinophyceae) from de Mexican Pacific. Acta Bot Mex 2006; 74:1-154.

23.- Licea-Durán S, Zamudio ME, Luna R, Soto J. Free-living dinoflagellates in the southern Gulf of México: Report of data (1979-2002). Phycol Res 2004; 52: 419-28.

24.- Hernández-Becerril DU, Almazán-Becerril A. Especies de dinoflagelados del género Gambierdiscus (Dinophyceae) del Mar Caribe mexicano. Rev Biol Trop 2004; 52 (Suppl.1):7787.

25.- Luckas B, Dahlmann J, Erler K, Gerdts G, Wasmund N, Hummert C. Hansen PD. Overview of key phytoplankton toxins and their recent occurrence in the North and Baltic Seas. Environ Toxicol 2005; 20:1-17.

26.- Rappala J, Robertson A, Negri AP, Berg KA, Tuomi P,
Lyra C, et al. First report of saxitoxin in Finnish lakes and possible associated effects on human health. Environ Toxicol 2005; 20: 331-40.

27.- Blanco-Pérez J. Episodios nocivos por fitoplancton. En Maeda-Martínez AN. editor. Los Moluscos Pectínidos de Iberoamérica: Ciencia y Acuicultura. México: Editorial Limusa; 2001. p. 285-324.

28.- Landsber JH. The Effects of Harmful Algal Blooms on Aquatic Organism. Rev Fish Sci 2002; 10:113-390.

29.- Acres J, Gray J. Paralytic shellfish poisoning. Can Med Assoc J 1978; 119:1195-7.

30.- Mata L, Abarca G, Marranghello L, Viquez R. Paralytic shellfish poisoning by Spondylus calcifer contaminated with Pyrodinium bahamense, Costa Rica 1989-1990. Instituto de Investigaciones en Salud (INISA), Universidad de Costa Rica.

31.- Hallegraeff GM. Harmful algal blooms: a global overview. En: Hallegraeff GM, Anderson DM, Cembella A, Editores. Manual on Harmful Marine Microalgae. Paris: UNESCO; 2003. p. 25-49.

32.- Chou HN, Chung YC, Chen CY. Evidence of paralytic shellfish poisoning toxin in milkfish in South Taiwan. Food Additives and Contaminants 2003; 20; 560-6.

33.- Goonetilleke A, Harris JB. Envenomation and consumption of poisonous seafood. J Neurol Neurosurg Psychiatry 2002; 73: 103-9.

34.- Bricelj MV, Shumway SE. Paralytic shellfish toxins in bivalve mollusks: occurrence, transfer kinetic, and biotransformation. Rev Fish Sci 1998; 6: 315-83.

35.- Mons MN, Van Egmond HP, Speijers GJA. Paralytic shellfish poisoning: A review. RIVM Report 388802 005. June 1998.

36.- Kulagina NV, O'Shaughnessy TJ, Ma W, Ramsdell JS, Pancrazio JJ. Pharmacological effects of the marine toxins, brevetoxin and saxiton, on murine frontal cortex neuronal networks. Toxicon 2004; 42:669-76.

37.- Stafford RG, Hines HB. Urinary elimination of saxitoxin after intravenous injection. Toxicon 1995; 53:1501-10.

38.- Andrinolo D, Michea LF, Lagos N. Toxic effects, pharmacokinetics and clearance of saxitoxin, a component of paralytic shellfish poison (PSP), in cats. 


\section{Hernández-Orozco, I Gárate-Lizárraga.}

Toxicon 1999; 37: 447-64.

39.- Shimizu Y. Chemistry and Mechanism of Action. En: Botana L, Dekker M, editores. Seafood and Freswater toxins Pharmacology, Phisiology, and Deteccion. Marcel Dekker Inc. New York: 2000, p 151-72.

40.- Schantz EJ. Chemistry and biology of saxitoxin and related toxins. Ann N Y Acad Sci 1986; 47: 15-23.

41.- Norma Oficial Mexicana de Emergencia, NOM-EM-005SSA1-2001, Salud Ambiental. Especificaciones sanitarias para el control de los moluscos bivalvos expuestos a la marea roja. Criterios para proteger la salud de la población.

42.- Hungerford JM. Committee on Natural Toxins and Food Allergens. Marine and freshwater toxins. JAOAC Int 2005; 88:299-313.

43.- Kerr DS, Briggs DM, Saba HI. A neurophysiological method of rapid detection and analysis of marine algal toxins. Toxicon 1999; 37: 1803-25.

44.- Sar EA, Ferrario ME, Reguera B editores: Floraciones Algales Nocivas en el Cono Sur Americano. Madrid: Instituto Español de Oceanografía; 2002.

45.- Van Egmond HP, Jonker KM, Poelman M, Scherpenisse P, Stern AG, Wezenbeek P, Bergwerff AA, Van den Top HJ. Proeficiency studies on the determination of paralytic shellfish poisoning toxins in shellfish. Food Addit Contam. 2004; $21: 331-40$.

46.- Vélez P, Sierralta J, Alcayaga C, Fonseca M, Loyola H, Johns DC, et al. A functional assay for paralytic shellfish toxins that uses recombinant sodium channels. Toxicon 2001; 39: 929-35.

47.- Louzao MC, Vieytes MR, Baptista de Sousa JM, Leira F, Botana LM. A fluorimetric method based on changes in membrane potential for screening paralytic shellfish toxins in mussels. Anal Biochem 2001; 289: 246-50.

48.- Powell CL, Doucette GJ. A receptor binding assay for paralytic shellfish poisoning toxins: recent advances and applications. Nat Toxins. 1999; 7: 393-400.

49.- Usleber E, Dietrich R, Burk C, Schneider E, Martbauer E. Immunoassay methods for paralytic shellfish posoning toxins. J AOAC Int. 2001; 84:1649-56.

50.- Laycock MV, Kralovec J, Richards R. some in vitro chemical interconversions of paralytic shellfish poisoning
(PSP) toxins useful in the preparation of analytical standards. J Mar Biotechnol 1995; 3:121-5.

51.- Wu JY, Zheng L, Wang JH. Contamination of shellfish from Shangai seafood markets with paralytic shellfish poisoning and diarrhetic shellfish poisoning toxins determined by mouse bioassay and HPLC. Food Additives and Contaminats 2005; 22: 647-51.

52.- Okumura $\mathrm{M}$, Tsuzuki $\mathrm{H}$, Tomita $\mathrm{B}$. A rapid detection meted for paralytic shellfish poisoning toxins by cell bioassay. Toxicon 2005; 46: 93-8.

53.- Kim CJ, Kim CH, Sako Y. Development of molecular identification method for genus Alexandrium (Dinophyceae) using whole-cell FISH. Marien Biotechnology 2005; 7: 21522.

54.- Position of the American Dietetic Association: Food and water safety. J Am Diet Assoc. 2003; 103: 1203-18.

55.- Lipp EK, Rose JB. The role of seafood in foodborne disease in the United States of America. Rev Sci Tech. 1997; 16: 620-40.

56.- Geraci JR, Anderson DM, Timperi RJ, St. Aubin DJ, Early GA, Prescott JH, et al. Humpback whales fatally poisoned by dinoflagellate toxin. Can J Fish Res Aquat Sci 1989; 46: 1895-8.

57.- Backer LC, Flemming LE, Rowan AD, Baden DG. Epidemiology, public health and human diseases associated with harmful marine algae. En: Hallegraeff GM, Anderson DM, Cembella A, Editores. Manual on Harmful Marine Microalgae. Paris: UNESCO; 2003. p. 723-749.

58.- Fleming LE, Baden D. Florida Red Tide and Human Health: Background. NIEHS Marine and Freshwater Biomedical Center, University of Miami 1999. (avalaible from: URL:htpp://www.redtide.whoi.edu/hab/illness/ floridaredtide.html)

59.- Rodrigue DC, Tzel RA, Hall S, de Porras E, Velásquez $\mathrm{OH}$, Tauxe RV, et al. Letal paralytic shellfish poisoning in Guatemala. Am J Trop Med Hyg 1990; 42: 267-71.

60.- Gessner BD, Middaugh JP. Paralytic shellfish poisoning in Alaska: a 20-year retrospective analysis. Am J Epidemiol 1995; 141:766-70.

61.- Gessner BD, Schloss M. A population-based study of paralytic shellfish poisoning in Alaska. Alaska Med 1996; 38: 54-68. 


\section{Envenenamiento paralizante por consumo de moluscos.}

62.- De la Garza-Aguilar J. Intoxicación alimentaria por ingestión de mariscos contaminados. Salud Pública Mex 1983; 25 : 145-50.

63.- Mee LD, Espinosa M, Diaz G. Paralytic Shellfish Poisoning with a Gymnodinium catenatum Red Tide on the Pacific Coast of Mexico. Marine Environmental Research 1986; 19: 77-92.

64.- Saldate-Castañeda O, Vázquez-Castellanos JL, Galván J, Sánchez-Anguiano A, Nazar A. Intoxicaciones por toxina paralizante de molusco en Oaxaca. Salud Pública Mex 1991; 33:240-7.

65.- Ronsón-Paulín, JA. Análisis retrospectivo y posibles causas de mareas rojas tóxicas en el litoral del sureste mexicano (Guerrero, Oaxaca, Chiapas). Ciencias del Mar. 1999; 9: 40-56.

66.- Cortés-Lara MC. Informe del fenómeno de marea roja en Bahía Banderas Jalisco-Nayarit, octubre- noviembre 2001. Rev Biomed 2002; 13: 73-5.

67.- Cortés-Altamirano R, Muñoz-Cabrera L, SotomayorNavarro O. Envenenamiento Paralítico por mariscos (PSP), causado por el dinoflagelado Pyrodinium bahamense var. compressum en la costa suroeste de México. Anales del Instituto de Ciencias del Mar y Limnología 1993; 20: 43-54.

68.- Cortés Altamirano R, Alonso Rodríguez R. Mareas rojas por Gymnodinium catenatum en Mazatlán 1997. Ciencias del Mar UAS 1999; 15: 31-7.

69.- Sierra-Beltrán AP, Cruz A, Núñez E, Del Villar M, Cerero $\mathrm{J}$, Ochoa JL. An overview of the marine food poisoning in México. Toxicon 1998; 36: 1493-502.

70.- Gárate-Lizárraga I, Bustillos-Guzmán JJ, AlonsoRodríguez R, Luckas B. Comparative paralytic shellfish toxin profiles in two marine bivalves during outbreaks of Gymnodinium catenatum (Dinophyceae) in the Gulf of California. Marine Pollution Bulletin 2004; 48: 397-402.

71.- Ochoa JL, Hernández-Becerril DU, Lluch-Cota S, Arredondo-Vega BO, Núñez-Vázquez E, Heredia-Tapia A, et al. Marine biotoxins and harmgul algal blooms in Mexico's Pacific litoral En: Taylor M, Trainer VL, editores. Harmful algal blooms in the PICES region of the North Pacific. PICES Scientific Report N²3 North Pacific Scie. 2002.

72.- Franco JM, Fernández-Villa P. Separation of Paralytic Shellfish toxins by reverserd phase high performance liquid chromatography, with postcolumn reaction and fluorimetric detection. Cromatographia 1993; 35: 613-20.

73.- Hay BE, Grant CM, McCoubrey DJ. A Review of the marine biotoxin monitorin programme for non-commercially harvested shellfish. Part 1: Technical report. A report prepared for the NZ Ministry of Health by AquaBio Consultants Ltd. NZ Ministry of Health 2000.

74.- Baden GD, Fleming LE, Bean JA. Marine Toxins. En: De Wolff FA editor. Handbook of Clinical Neurology: Intoxications of the Nervous System. Amsterdan: Elsevier Publishing; 1995. p. 141-75.

75.- Luke Y, Dart RC. Food Poisoning-Shellfish. En: Dart RC editor. 5 minute toxicology Consultan. London: Lippincott, Williams \& Wilkins 2000. p. 392-3.

76.- Harris JB, Goonetilleke A. Animal poisons and the nervous system: what the neurologist needs to know. J Neurol Neurosurg Psychiatry 2004; 75 (Suppl III) iii40-iii46.

77.- Gessner BD, Bell P, Doucette GJ, Mocczydlowski E, Poli MA, Van Dolah F, Hall S. Hypertension and identification of toxin in human urine and serum following a cluster o musselassociated paralytic shellfish poisoning outbreaks. Toxicon 1997; 35: 711-22.

78.- Wallace BJ, Guzewich JJ, Cambridge M, Altekruse S, Morse DL. Seafood-Associated Disease Outbreaks in New York, 1980-1994. Am J Prev Med 1999; 17: 48-54.

79.- De Carvalho M, Jacinto J, Ramos N, De Oliveira V, Pinho T, Joao de Sá M. Paralytic shellfish poisoning:clinical and electrophysiological observations. J Neurol 1998; 245: 5514.

80.- Long RR, Sargent JC, Hammer K. Paralytic shellfish poisoning: a case report and serial electrophysiologic observations. Neurology 1990; 40: 1310-2.

81.- Cheng HS, Chua SO, Hung JS, Yip KK. Creatin kinase MB elevation in paralytic shellfish poisoning. Chest 1991; 99: 1032-3.

82.- Mahmud Y, Arakawa O, Noguchi T. An epidemic survey on freswater puffer poisoning in Bangladesh. J Nat Toxins 2000; $9 ; 319-26$.

83.- Isbister GK, Kiernan MC. Neurotoxic marine poisoning. Lancet Neurology 2005; 4: 219-28.

84.- Negoro K, Morimatsu M. Clinical analysis of paralytic shellfish poisoning following ingestion of oysters. Rinsho 


\section{Hernández-Orozco, I Gárate-Lizárraga.}

Shinkeigaku 1993; 33: 207-9.

85.- Kan SK, Singh N, Chan MK. Oliva vidua fulminans, a marine mollusk, responsible for five fatal cases of neurotoxic food poisoning in Sabah, Malaysia. Trans R Soc Trop Med Hyg 1986; 80:64-5.

86.- García C, Bravo MC, Lagos M, Lagos N. Paralytic shellfish poisoning: post-mortem analysis of tissue and body fluid samples from human victims in the Patagonia fjords. Toxicon 2004; 43: 149-58.

87.- Duncan KG, Duncan JL, Schwartz DM. Saxitoxin: An anesthetic of the deepithelialized rabbit cornea. Cornea 2001; 20: 639-42.

88.- Montebruno D. Poisoning by the consumption of shellfish contaminated with paralytic venom in the XII Region, Chile. Anatomopathological study. Rev Med Chil 1993; 121:94-7.

89.- Garrido R, Lagos N, Lattes K, Azolas CG, Bocic G, Cuneo $\mathrm{A}$, et al. The gonyautoxin $2 / 3$ epimer reduces anal tone when inyected in the anal sphincter of healthy adults. Biol Res 2004; 37: 395-403.

90.- Graham HW. Gymnodinium catenatum a new dinoflagellate from the Gulf of California. Trans Am Microscop Soc 1943; 62: 259-61.

91.- Sierra-Beltrán AP, Morquecho-Escamilla ML, LechugaDevéze C, Ochoa JL. PSP monitoring program at Baja California Sur, Mexico. En Yasumoto T, Oshima Y, Fukuyo Y, editores. Harmful and Toxic Algal Blooms. Intergovernmental Oceanographic Commission of UNESCO, Paris. 1996. p. 1058.

92.- Orellana-Cepeda E, Martínez-Romero E, Muñoz-Cabrera L, López-Ramírez P, Cabrera-Mancilla E, Ramírez-Camarena C. Toxicity associated with blooms of Pyridinium bahamense var. compressum in south western México. En: Reguera B, Blanco J, Fernández M L, Wyatt T, editores. Harmful Algae. Xunta de Galicia, IOC 1998. p. 60.

93.- Gárate-Lizárraga I, Hernández-Orozco ML, Band-Schmidt C, Serrano-Casillas G. Red tides along the coasts of Baja California Sur, México (1984 to 2001). Oceánides 2001; 16: 127-34.

94.- Gárate-Lizárraga I, Bustillos-Guzmán JJ, Erler K, Muñeton-Gómez MS, Luckas B. Tripp-Quezada A. Paralytic shellfish toxins in the chocolata clam, Megapitaria squalida (Bivalvia: Veneridae), in Bahía de La Paz, Gulf of California.
Rev Biol Trop 2004; 52: 133-40.

95.- Band-Schmidt CJ, Bustillos-Guzmán J, Gárate-Lizárraga I, Lechuga-Devéze CH, Reinhardt K, Luckas B. Profiles of paralytic shellfish toxin in strains of the dinoflagellate Gymnodinium catenatum and the scallop Argopecten ventricosus from Bahía Concepción, Gulf of California, Mexico. Harmful Algae 2005; 4: 21-31.

96.- Gárate-Lizárraga I, Bustillos-Guzmán JJ, Morquecho M, Band-Schmidt CJ, Alonso-Rodriguez R, Erler K, et al. Comparative paralytic shellfish toxin profiles in the strains of Gymnodinium catenatum Graham from the Gulf of California, Mexico. Marine Pollution Bulletin 2005; 50: 2139.

97.- Cifuentes-Lemus J.L, Torres-García P, Frías-M M. El Océano y La pesca. IX. La Pesca. México: Fondo de Cultura Económica; Segunda Edición 1997. pp. 125.

98.- Cifuentes-Lemus J.L, Torres-García P, Frías-M M. El Océano y sus recursos $\mathrm{X}$. Las pesquerías. Fondo de Cultura Económica, Segunda Edición, México, D.F. 1997 pp 130.

99.- Whittle K Gallacher S. Marine toxins Health and the food-chain. Br Med Bull 2000; 56:236-53.

100.- Bicelj VM, Connell L, Konoki K, Macquarrie SP, Scheuer T, Catterall WA, Trainer VL. Nature 2005; 434:716.

101.- Cortés-Altamirano, RA, Sierra-Beltrán P., Cortés-Lara M. C. 2004. Dominance and permanence of species of harmful algae forming blooms in Mazatlán Bay, Mexico (1979-2002). En: Steidinger, K. A., J. H. Landsberg, C. R. Tomas \& G. A. Vargo, editors. Harmful Algae 2002. Xth International Conference, St. Pete Beach, Florida, USA, October 21-25, 2002. Florida Fish and Wildlife Conservation Commission, Florida Institute of Oceanography, and Intergovernmental Oceanographic Commission of UNESCO; 2004. p. 344-6.

\section{Revista Biomédica}

